\title{
Ultralow Threshold and Single-Mode Lasing in Microgear Lasers and Its Fusion With Quasi-Periodic Photonic Crystals
}

\author{
Kengo Nozaki, Atsuo Nakagawa, Daisuke Sano, and Toshihiko Baba, Member, IEEE
}

\begin{abstract}
The GaInAsP microgear laser is a kind of microdisk laser but has a rotationally symmetric grating, which matches with the profile of the whispering gallery mode. In this study, we achieved the room temperature continuous-wave lasing with a threshold photopump power of $14 \mu \mathrm{W}$ for the smallest gear diameter of $2.02 \mu \mathrm{m}$. We also observed the single-mode lasing in a deep grating device, where the $\pi$-phase-shifted nonlasing mode completely disappeared. The experimental results were well explained by a three-dimensional finite-difference time-domain calculation and a consideration on the surface recombination. In addition, to increase the variety of the microgear, we proposed its fusion with a quasi-periodic photonic crystal (QPC), which also has a rotational symmetry. We theoretically estimated a sufficiently high $Q$ of 21600 for a QPC gear with a point defect of 1.6- $\mu \mathrm{m}$ diameter, which is the smallest limit for a normal microdisk at a wavelength of 1.5-1.6 $\mu \mathrm{m}$.
\end{abstract}

Index Terms-GaInAsP/InP, microdisk, microgear, photonic crystal (PC), point defect laser, quasi-periodic photonic crystal (QPC), semiconductor laser, whispering gallery mode (WGM).

\section{INTRODUCTION}

$\mathbf{M}$ ICRODISKS, [1]-[4] microrings, [5] microcylinders, [6], [7], and so forth are semiconductor microlasers that operate by whispering gallery modes (WGMs). Particularly, microdisk lasers have a high $Q$ due to their simple structure and strong optical confinement by the total internal reflection at semiconductor/air boundaries. Furthermore, in microdisks with compressively-strained quantum-well (CS-QW) active layer, the effective carrier confinement is achieved by the strain-relaxation effect [8]. Because of such effects, ultralow threshold lasings have been achieved. In our previous studies, we fabricated 1.5-1.6- $\mu \mathrm{m}$ wavelength range GaInAsP-InP CS-QW devices, and obtained a threshold current of $40 \mu \mathrm{A}$ [9] and a threshold power of $30 \mu \mathrm{W}$ [10] in a current injection device and in a photopumped device, respectively, at room temperature under continuous wave (CW) condition. We also evaluated a large spontaneous emission factor, which is a unique property of microlasers suggesting the possibility of the thresholdless lasing [10]-[14]. However, when the cavity size is reduced to close to the diffraction limit of the WGM,

Manuscript received February 18, 2003; revised July 22, 2003. This work was supported by the Grant-in Aid 13305009, IT Project, and 21st COE Program, all from the Ministry of Education, Culture, Sports, Science and Technology. It was also supported by CREST 530-13 of Japan Science and Technology Corporation.

The authors are with the Department of Electrical and Computer Engineering, Yokohama National University, 79-5 Tokiwadai, Hodogayaku, Yokohama 2408501, Japan (e-mail: baba@ynu.ac.jp).

Digital Object Identifier 10.1109/JSTQE.2003.819465

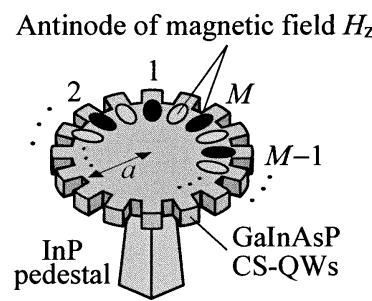

(a)

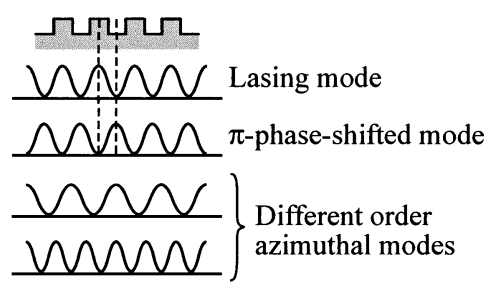

(b)
Fig. 1. Schematics of microgear laser and resonant modes. (a) Microgear laser with $H_{z}$ standing wave of WG mode matched with the grating. $M$ is the azimuthal mode order. (b) $\left|H_{z}\right|^{2}$ of resonant modes.

i.e., $\sim 1.6 \mu \mathrm{m}$ diameter, the $Q$ factor is seriously degraded. Moreover, this device often exhibits a nonlasing mode very close to the lasing mode. This mode is thought to have a $\pi$-phase-shifted mode profile against the lasing mode profile. Such a mode is undesirable for the reduction in threshold and for the increase in spontaneous emission factor.

The microgear laser has a grating at the disk edge with a period equal to the half wavelength of the WGM [15], [16], as shown in Fig. 1. The two-dimensional (2-D) finite-difference time-domain (FDTD) analysis with the equivalent index approximation explained the strong optical confinement and the single-mode operation as follows [13]. Let us assume here that only TE-polarized modes are allowed due to the electron transition between the conduction band and the heavy-hole valence band in CS-QWs. Then, the normal component of the magnetic field $H_{\mathrm{Z}}$ and the radial component of the electric field $E_{\mathrm{r}}$ generate the WGM energy, while $H_{\mathrm{z}}$ and the azimuthal component of the electric field $E_{\theta}$ generate the radiation power to outside of the cavity. $E_{\mathrm{r}}$ is discontinuous at the disk boundary, so it penetrates into air more deeply than $H_{\mathrm{z}}$. To compensate for this difference, $E_{\theta}$ is excited, and this results in radiation loss. In microgear lasers, penetration depths of $E_{\mathrm{r}}$ and $H_{\mathrm{z}}$ are equalized by the grating. Therefore, the generation of $E_{\theta}$ is suppressed, and the $Q$ factor is increased. That is to say, it is possible to reduce the cavity size more than ever. In addition, $E_{\theta}$ of the $\pi$-phase-shifted mode is more excited and its $Q$ factor is reduced. Thus, this mode is suppressed and the stable single mode is realized.

Previously, we obtained the lasing operation with an effective threshold power of $21 \mu \mathrm{W}$ in a $2.7-\mu \mathrm{m}$-diameter (inner diameter) GaInAsP-InP microgear laser [16]. (The claimed value in [16] was $17 \mu \mathrm{W}$, but its evaluation was corrected in this study, as explained later.) However, there still remains room for the reduction in size and threshold. Besides, since the 


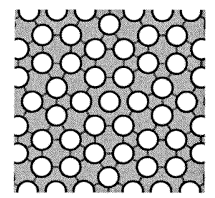

(a)

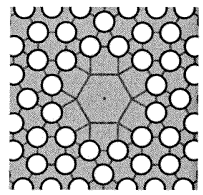

(b)

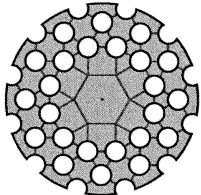

(c)

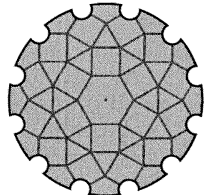

(d)

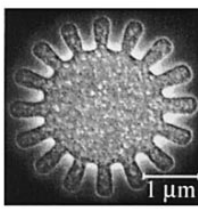

(a)

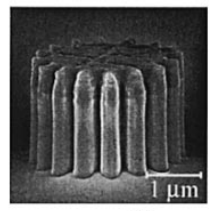

(b)

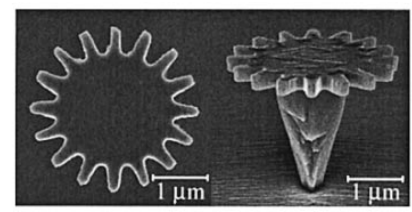

(c)
Fig. 2. Schematic of a fusion of QPC and microgear. (a) 12-fold symmetric QPC. (b) QPC cavity with a point defect. (c) QPC gear. (d) Microgear.

grating in the device was relatively shallow, the suppression of the $\pi$-phase-shifted mode was not clear. In this paper, we first describe the improvement of the fabrication process and show some lasing characteristics exhibiting a lower threshold and clearer single-mode operation. Then, we present a three-dimensional (3-D) FDTD calculation, which was carried out in order to verify the above discussion based on the previous 2-D calculation. We will show that the result explains the experiment result very well.

In this paper, we also propose the fusion of a quasi-periodic photonic crystal (QPC) [17]-[19] and the microgear to overcome the size limit. The QPC has a higher order rotational symmetry, as shown in Fig. 2(a). Its 2-D photonic bandgap (PBG), similar to that in a standard photonic crystal (PC), has been demonstrated theoretically and experimentally. The QPC inside the microgear laser, as shown in Fig. 2(c), is expected to strengthen the confinement of the WGM and allow a smaller mode volume. In this paper, we show transmission and resonant characteristics of a QPC and its point-defect cavity, which were calculated by the 2-D FDTD method. Moreover, we show resonant characteristics of a more realistic QPC gear cavity by the 3-D FDTD method.

\section{MICROGEAR LASERS}

\section{A. Fabrication Process and Lasing Characteristics}

In this experiment, we prepared a GaInAsP-InP epitaxial wafer with five CS-QWs of 4-nm thickness each, $1 \%$ strain, and $1.58-\mu \mathrm{m}$ photoluminescence peak (this peak was redshifted after the disk formation by the strain relaxation [8]). In addition to the CS-QWs, the active layer included 1.2- $\mu \mathrm{m}$-quaternary (Q) barrier layers of 10-nm thickness, and 1.2-, 1.15-, and 1.1-Q separate confinement heterostructure layers, each of which had a $30 \mathrm{~nm}$ thickness. Thus, the total thickness of the active layer was $\sim 240 \mathrm{~nm}$. First, we evaporated Ti film on the substrate as a mask, and formed a resist pattern by electron beam (EB) lithography, as shown in Fig. 3(a). Here, we used a data grid of $10 \mathrm{~nm}$ in the EB computer-aided design system, which was 2.5 times finer than before. This significantly improved the uniformity and symmetry of the grating. Next, we formed a $\mathrm{Cr}$ pattern by the evaporation and the liftoff technique, and transferred it to $\mathrm{Ti}$ using $\mathrm{CF}_{4}$ dry etching. The microgear mesa was formed by $\mathrm{Cl}_{2} / \mathrm{Xe} \mathrm{ICP}$ etching. Conditions of these etchings were the same as those reported previously [10], [16]. As shown in Fig. 3(b), the mesa had almost vertical and smooth sidewalls. Finally, the Ti mask was removed by HF, the microgear disk was formed by the selective wet etching of the InP cladding using an $\mathrm{HCl}$ solution, as shown in Fig. 3(c).

In the measurement, each device was photopumped continuously by $0.98-\mu \mathrm{m}$ semiconductor laser light through a lens
Fig. 3. Scanning electron micrographs of devices after each fabrication process. (a) Resist pattern by EB lithography. (b) Microgear mesa formed by $\mathrm{Cl}_{2} / \mathrm{Xe} \mathrm{ICP}$ etching. (c) Microgear disk formed by $\mathrm{HCl}$ wet etching.

system at room temperature $\left(20^{\circ} \mathrm{C}-22{ }^{\circ} \mathrm{C}\right)$. The emitted light was detected by a multimode fiber whose tip was placed $\sim$ $100 \mu \mathrm{m}$ apart from the device and $30^{\circ}$ inclined against the substrate plane, and then analyzed by optical spectrum analyzer. Using the input power $P_{0}$ and the intensity distribution of the irradiated light $P(r)$ on the substrate plane, the irradiated power to the device $P_{1}$ was calculated by the following:

$$
P_{1}=P_{0} \cdot \frac{\int_{0}^{2 \pi} \int_{0}^{a} r P(r) d r d \theta}{\int_{0}^{2 \pi} \int_{0}^{\infty} r P(r) d r d \theta}
$$

where $a$ is the gear radius and $r$ is the radius from the center. The effective pump power (absorbed power) to the device $P_{2}$ is expressed by taking account of a resonant absorption inside the disk as

$$
\begin{gathered}
P_{2}=P_{1}\left\{(1-R)\left(1-e^{-\alpha d}\right)+R e^{-\alpha d}(1-R)\left(1-e^{-\alpha d}\right)\right. \\
\left.\quad+R^{2} e^{-2 \alpha d}(1-R)\left(1-e^{-\alpha d}\right)+\cdots\right\} \\
=P_{1} \frac{(1-R)\left(1-e^{-\alpha d}\right)}{1-R e^{-\alpha d}}
\end{gathered}
$$

where $R$ is the reflectivity at disk surfaces $(\sim 30 \%), \alpha$ is the absorption coefficient of the disk $\left(\sim 2 \times 10^{4} \mathrm{~cm}^{-1}\right)$, and $d$ is the disk thickness $(\sim 240 \mathrm{~nm})$. (In our previous papers, we calculated $P_{2}$ without considering the resonance absorption and used only the first term of (2). Equation (3) indicates that the power should be corrected by factor $1 /\left(1-R e^{-\alpha d}\right)$, which is calculated to be 1.23 for the above parameters.)

Fig. 4(a) shows the lasing characteristics in a device, which exhibited the lowest threshold. The device had an inner diameter of $2.02 \mu \mathrm{m}$, a grating number of 16 , and a grating depth of 90-110 nm (there were some nonuniformities). The threshold effective pump power $P_{2}$ is estimated to be $14 \mu \mathrm{W}$ (irradiated power $P_{1}$ of $\left.42 \mu \mathrm{W}\right)$. This is nearly $67 \%$ of that obtained previously [16]. The corresponding threshold power density is $380 \mathrm{~W} / \mathrm{cm}^{2}$ or $76 \mathrm{~W} / \mathrm{cm}^{2}$ - well. This value is much lower than a threshold power density of $\sim 700 \mathrm{~W} / \mathrm{cm}^{2}$ of the epitaxial wafer, which was estimated by converting the threshold current density ( $\sim 800 \mathrm{~A} / \mathrm{cm}^{2}$ for long broad area lasers of this wafer) into power dimension. In the spectral characteristics, the lasing mode peak at $P_{2}=34 \mu \mathrm{W}$ was $35 \mathrm{~dB}$ higher than the background spontaneous emission level, and the full-width at half-maximum (FWHM) was $0.3 \mathrm{~nm}$, the resolution limit determined by the used multimode fiber for detection and by the optical spectrum analyzer. The lasing wavelength was 1.655 $\mu \mathrm{m}$, which was much longer than the wafer gain peak due to the above-mentioned strain relaxation effect. The lasing mode is considered to be the matching mode with the grating. Spectral characteristics in Fig. 4(a) also show another strong intensity mode with 3-nm shorter wavelength. This nonlasing mode 


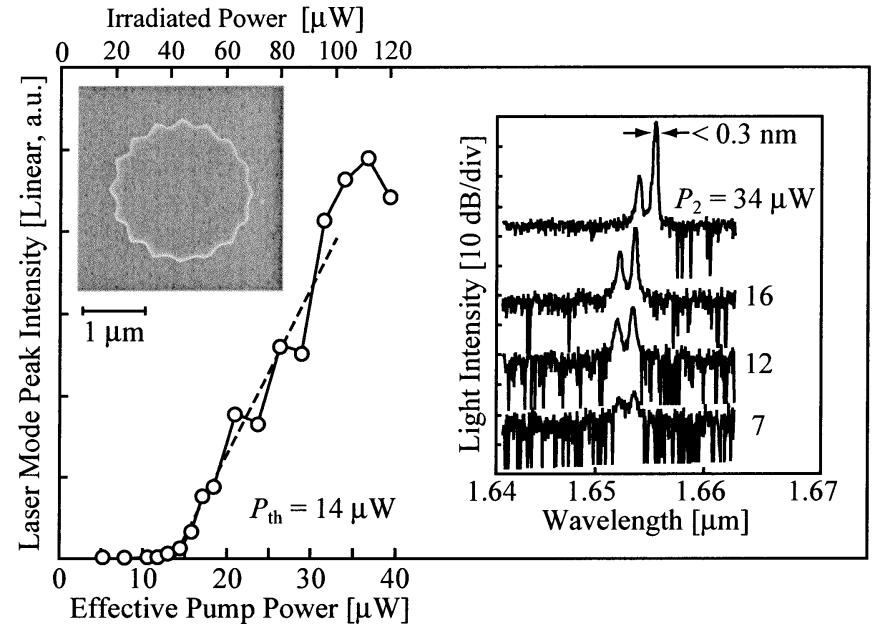

(a)

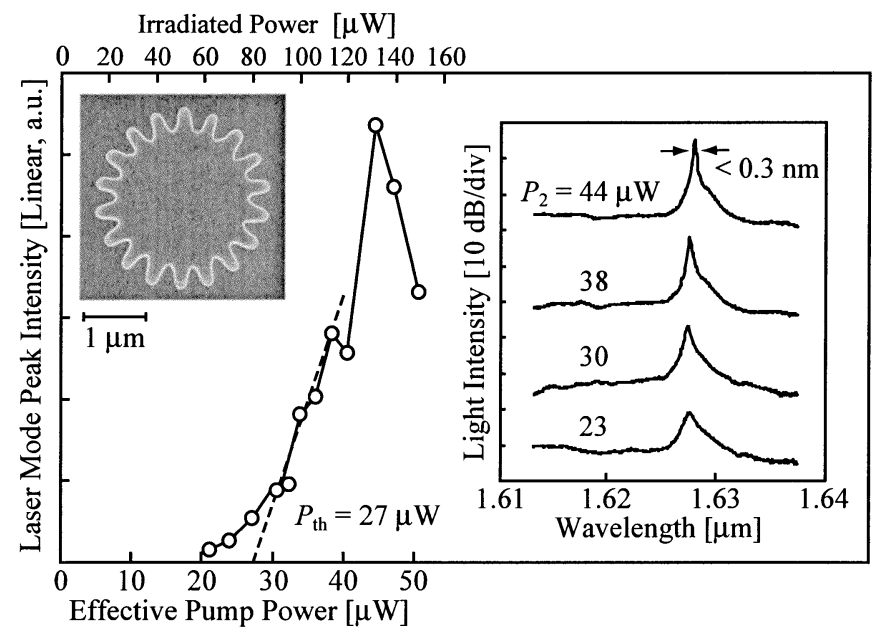

(b)

Fig. 4. Lasing characteristics at room temperature by $\mathrm{CW}$ photopumping (a) Shallow grating device. (b) Deep grating device.

should be the $\pi$-phase-shifted mode. The weak suppression of this mode is considered to be due to the shallow grating [15].

Fig. 4(b) shows lasing characteristics of another device with an inner diameter of $2.12 \mu \mathrm{m}$, a grating number of 18 and a deeper grating depth of $360 \mathrm{~nm}$. Unlike that for the shallow grating device, this device exhibited the clear single-mode lasing with a slightly broad linewidth at lower intensity level. The threshold power $P_{2}$ of this device was $27 \mu \mathrm{W}\left(P_{1}=82 \mu \mathrm{W}\right)$, which was twice that of (a).

\section{B. Theoretical Analysis}

The spectral characteristics in Section II-A showed the dependency on the grating depth. Such a dependency was not only observed for a limited number of devices, but also observed for many devices. In order to explain these characteristics, we calculated WGMs using the 3-D FDTD method. To model the shape of the lasing devices as true as possible, the inner diameter was set to be 2.10 or $2.16 \mu \mathrm{m}$, the grating number to be 16 or 18 , and the grating depth to be 90 or $360 \mathrm{~nm}$, respectively, for a cubic Yee cell of $(30-\mathrm{nm})^{3}$ size. The total thickness of the disk was set to be $240 \mathrm{~nm}$. QWs of $60 \mathrm{~nm}$ in total thickness were assumed at the center of the disk. As a refractive index of the QWs, we assumed a large value of 4.5. If the band edge of the QWs is flat, it may be more reasonable to use a lower index of about 3.5. However, since the lasing wavelength is determined by the WGM, the wavelength can shift from the band edge. In this case, the index sharply increases due to the material dispersion, so an index of 4.5 may be possible. In addition, spectra calculated by the FDTD method with an index of 4.5 well explained the experiment result, as shown later. Regarding other layers in the disk, we assumed indexes of 3.320, 3.292, and 3.265. Considering the crystal facet of the InP pedestal, which appeared after the $\mathrm{HCl}$ etching, we assumed a square cross-section of the pedestal with one side width of $540 \mathrm{~nm}$. Its height and index were assumed to be $0.6 \mu \mathrm{m}$ and 3.17 , respectively. The time interval between calculation steps was $0.025 \mathrm{fs}$, which satisfied the Courant's condition. The analytical space was $(5.4 \mu \mathrm{m})^{2}\left(=180^{2}\right.$ cells $)$ in the lateral direction, and $1.5 \mu \mathrm{m}(=50$ cells $)$ in the vertical direction. Mur's second-order absorbing condition was used as a boundary condition on the periphery of the analytical space. The excitation was given by a Gaussian pulse for the vertical component of the magnetic field $H_{z}$, considering the TE-polarized emission of the CS-QWs. The time step at the pulse peak was 5000. The center wavelength $\lambda$ and the full spectral width at $1 / e^{2}$ of the maximum intensity were $1.65 \mu \mathrm{m}$ and $0.1(2 \pi c / \lambda)$, respectively, where $c$ is the light velocity in vacuum. Under this condition, the spectrum covered the wavelength range of $1.55-1.75 \mu \mathrm{m}$. An excitation point was set close to the disk edge at a middle azimuthal angle between the convex and concave of the grating. Near the disk edge, the excitation was easily coupled with the WGM. The middle angle is effective for exciting arbitrary phase modes.

Fig. 5(a) and (b) shows calculated spectra for the devices with shallow and deep gratings, respectively. Here, the $H_{z}$ distribution is also shown for each resonant mode. Such distributions were calculated by selectively exciting each mode with a narrow spectral width of $0.04(2 \pi c / \lambda)$. In Fig. 5(a), the main peak at $\lambda=1.65 \mu \mathrm{m}$ should correspond to the lasing mode in the experiment, since antinodes of $H_{z}$ of this mode locate at convexes of the grating. Another peak at a slightly shorter wavelength should correspond to the $\pi$-phase-shifted mode. The other two peaks at the shortest and longest wavelengths, which were not clearly observed in the experiment, are mismatching modes with the grating. On the other hand, in Fig. 5(b), the $\pi$-phase-shifted mode is absolutely disappearing. This difference between (a) and (b) agrees with the experimental result in Fig. 3. Two possible reasons are considered for the difference. One is that the $\pi$-phase-shifted mode is suppressed by the enhanced mode selectivity of the deep grating. The other is that the $\pi$-phase-shifted mode is absolutely degenerated with the lasing mode. As observed in Fig. 5(b), the antinode of $H_{z}$ is located at a middle angle between the convex and the concave. When we placed the excitation point at either angle just at the convex or the concave, we observed the distribution of the main mode or the $\pi$-phase-shifted mode individually. These results imply that both modes exist simultaneously in the spectrum of Fig. 5(b). The experimental linewidth of the lasing mode at lower intensity level, as shown in Fig. 4(b) can be explained by such degenerate modes.

The dependency of the resonant wavelength of the matching and $\pi$-phase-shifted modes on the grating depth has been calculated previously by the 2-D FDTD method [16]. It indicated that the wavelength of the matching mode rapidly becomes longer in 


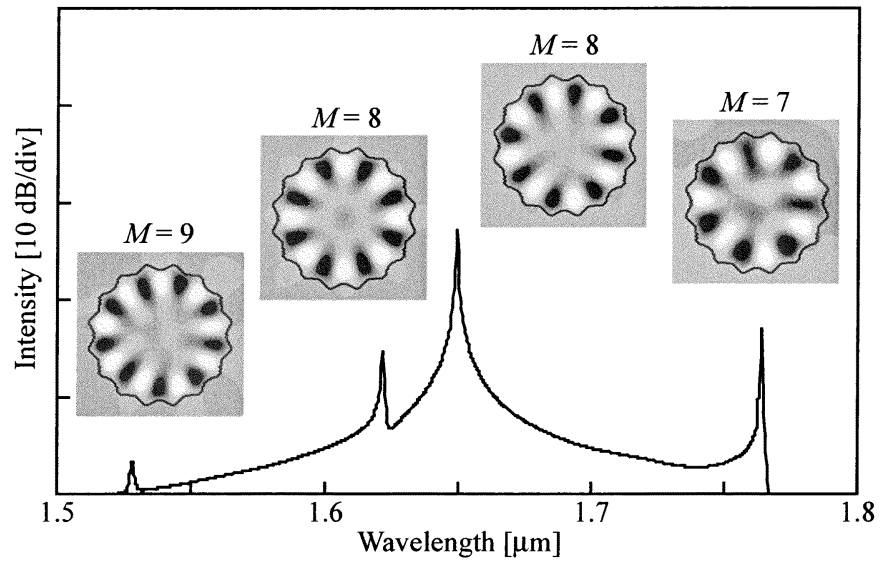

(a)

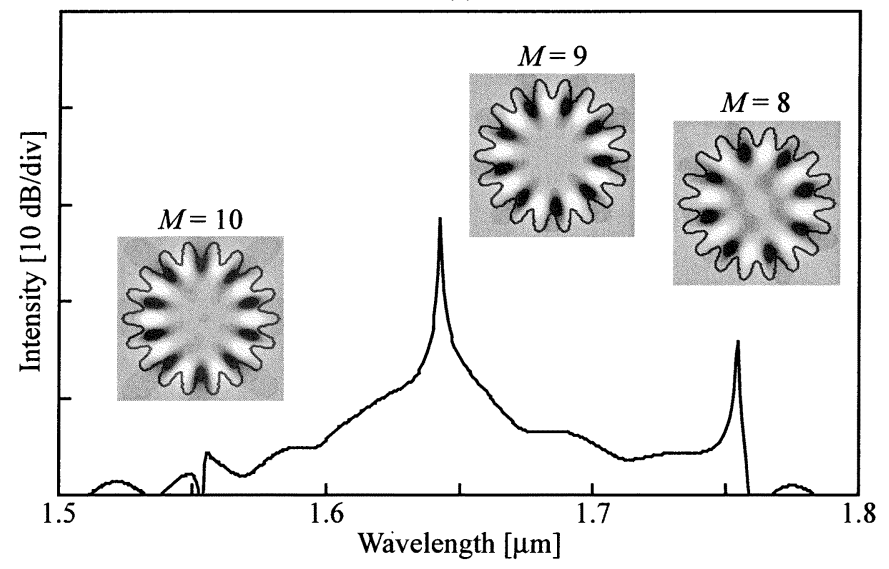

(b)

Fig. 5. Spectral characteristics and $H_{z}$ distribution of each mode peak, which are calculated by the 3-D FDTD method. (a) Shallow grating device. (b) Deep grating device.

a shallow grating regime but saturates in a deep grating regime, while the wavelength of the $\pi$-phase-shifted mode rapidly becomes longer in a deep grating regime. There is a cross-point for a grating depth of around $200 \mathrm{~nm}$. In this study, we performed the similar calculation by the 3-D FDTD, but the result was almost the same. When the grating depth is equal to or less than $200 \mathrm{~nm}$ (almost equal to the penetration depth of the evanescent wave), the orbital length of the $\pi$-phase-shifted mode is shortened by the grating, so its resonant wavelength becomes shorter than that of the matching mode. When the depth is larger than $200 \mathrm{~nm}$, the spreading of $E_{\mathrm{r}}$ to air is suppressed and the orbital length of the matching mode does not change so much, since antinodes of $E_{\mathrm{r}}$ of this mode are located at concaves of the grating. On the other hand, antinodes of $E_{\mathrm{r}}$ of the $\pi$-phase-shifted mode are located at convexes of the grating, so $E_{\mathrm{r}}$ can spread. Consequently, the orbital length of the mode increases and the wavelength becomes longer than the matching mode.

Next, let us consider the difference of threshold between the devices with different grating depths. As explained in Section I, the 2-D calculation showed that the radiation loss is caused by the excitation of $E_{\theta}$. Fig. 6 shows the distribution of each electromagnetic component of the main mode in a microdisk and the two microgears, which was calculated by the 3-D FDTD method. As shown in Fig. 6(a), $E_{\mathrm{r}}$ in the microdisk is discontinuous at the disk boundary and largely spreading to air, so a
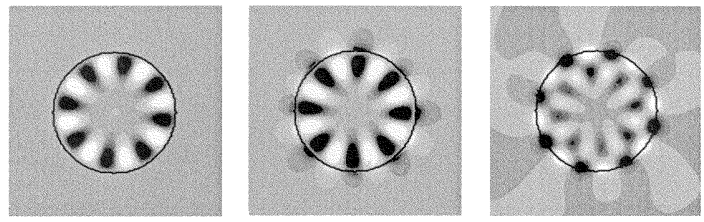

(a)
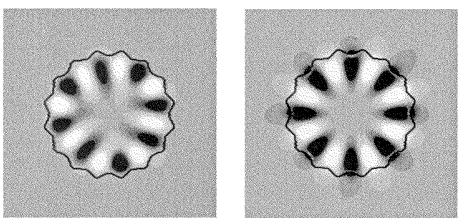

(b)
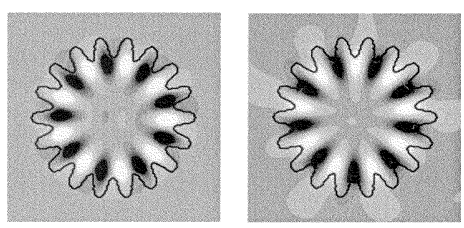

(c)
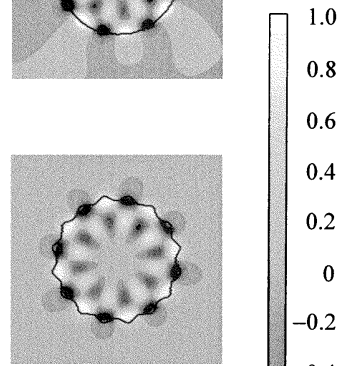

0.6

0.4

0.2

0

$-0.2$

$-0.4$

$-0.6$

$-0.8$

$-1.0$

Fig. 6. Distribution of each electromagnetic component of TE mode calculated by the 3-D FDTD method. Left, center and right are $H_{\mathrm{z}}, E_{\mathrm{r}}$, and $E_{\theta}$, respectively. (a) Microdisk with $2 a=2.16 \mu \mathrm{m}$. (b) Microgear with shallow grating. (c) Microgear with deep grating.

large mismatch between $E_{\mathrm{r}}$ and $H_{z}$ excites $E_{\theta}$. In the shallow grating gear, this mismatch is slightly reduced, but $E_{\theta}$ is still excited, as shown in Fig. 6(b). In the deep grating gear, the mismatch is further reduced and $E_{\theta}$ is more suppressed, as shown in Fig. 6(c). These results are almost the same as those by the $2-\mathrm{D}$ calculation. The ratio of the time-averaged radiation power to the stored mode energy indicated that the $Q$ factors are 3300 and 3600 for the shallow and deep grating gears, respectively. They are not so significantly different, but still we should expect a lower threshold for the deep grating device. However, it cannot explain the experimental result.

Another explanation considered is the change of the surface recombination between the two devices. For the same epitaxial wafer, we measured the radiative recombination lifetime of $\sim 10 \mathrm{~ns}$ at threshold [20] and the surface recombination velocity of $1.2 \times 10^{4} \mathrm{~cm} / \mathrm{s}$. [21] From these results, we found that, for a microdisk of less than $2.5 \mu \mathrm{m}$ in diameter, the surface recombination becomes a dominant factor for threshold. Differences of the sidewall area and the active volume between the two devices cannot be ignored in estimating the threshold. If the same $Q$ factor was assumed, the threshold for the shallow grating device was estimated to be the same as that for the microdisk, since the triangular-like shape grating of this device does not increase the sidewall area as such. On the other hand, the threshold for the deep grating device was estimated to be 1.9 times that of the shallow grating device. This value almost explains the experimental result.

\section{FUSION OF QPC AND MICROGEAR}

In this section, we discuss the QPC, as shown in Fig. 2(a), which is composed of holes in a high index background. It has 12-fold rotational and line symmetries, so the structure has good consistency to the WGM of the microgear. Let us also discuss a QPC cavity, as shown in Fig. 2(b), and the QPC gear, as shown 


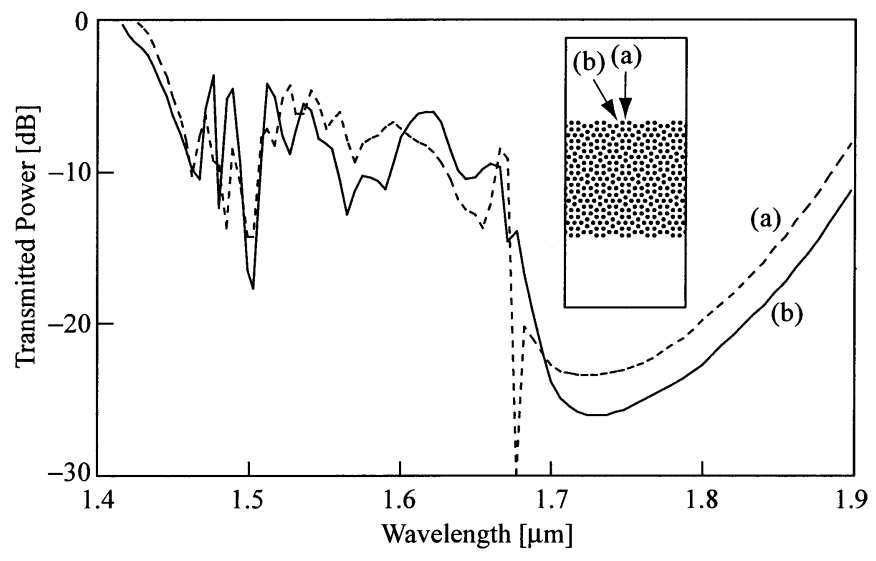

Fig. 7. Transmission spectra of QPC with 12 -fold symmetry calculated by the 2-D FDTD method. The inset shows the calculation model. (a) and (b) are those for the light incident angle of $0^{\circ}$ and $30^{\circ}$, respectively.

in Fig. 2(c). Here, the cavity is composed of a point defect of missing holes.

To confirm the PBG of the QPC, we first analyzed the transmission spectrum of the QPC by the 2-D FDTD method. The background index was assumed to be 2.65 (the typical equivalent index of the fundamental guided mode in the disk), the hole diameter to be $420 \mathrm{~nm}$, the lattice constant to be $585 \mathrm{~nm}$ and the number of holes to be 337. The size of Yee cell was $(15 \mathrm{~nm})^{2}$ and the time interval was $0.025 \mathrm{fs}$. The spectral width of the Gaussian excitation was $0.16(2 \pi c / \lambda)$, which covered the wavelength range of $1.50-1.80 \mu \mathrm{m}$. The excitation points were chosen so that a plane wave incident to the QPC with an incident angle of 0 or $30^{\circ}$ was excited. Fig. 7 shows calculated transmission spectra. A transmission suppression of over $20 \mathrm{~dB}$ is observed at the wavelength range of 1.67 to $1.80 \mu \mathrm{m}$ for both angles. This suggests the existence of a PBG.

Next, we calculated resonant characteristics in the QPC cavity by the 2-D FDTD method. In the calculation model, we assumed one or seven missing holes in the same QPC as that for Fig. 7. As shown in Fig. 8, clear resonant peaks and corresponding localized modes were observed in both cavities. The dipole modes in the single point defect in Fig. 8(a) look almost the same as those in the single defect photonic crystal cavity. [22] It is known that in a normal PC cavity, the splitting of two dipole modes occurs due to the break of mode degeneracy. Even though the splitting in Fig. 8(a) looks large, it is also arising from such a break of degeneracy in the calculation model with a small asymmetry. The $Q$ factor of this mode was calculated to be $\sim 9700$. In Fig. 8(b), A and B correspond to radial mode order 1 and 0 , and have $Q$ factors of $\sim 7000$ and $\sim 220000$, respectively. The high $Q$ for mode B is attributed to its standing wave well matching with the hole arrangement around the defect.

A real device will have a disk shape to realize the 3-D strong optical confinement. Therefore, we next calculated modal characteristics in a QPC gear cavity by the 3-D FDTD method. The thickness and the index of the disk, and FDTD parameters were the same as those for Fig. 5. The design of the QPC is the same as for Figs. 7 and 8. Fig. 9 shows resonant characteristics for the QPC gear with an outer disk diameter of $3.84 \mu \mathrm{m}$. Similarly to the case of the 2-D calculation, the mode is well con-

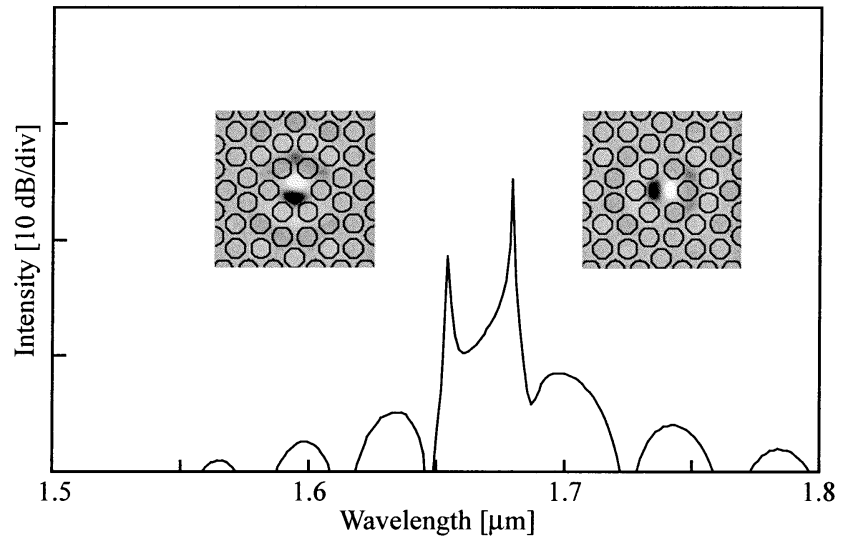

(a)

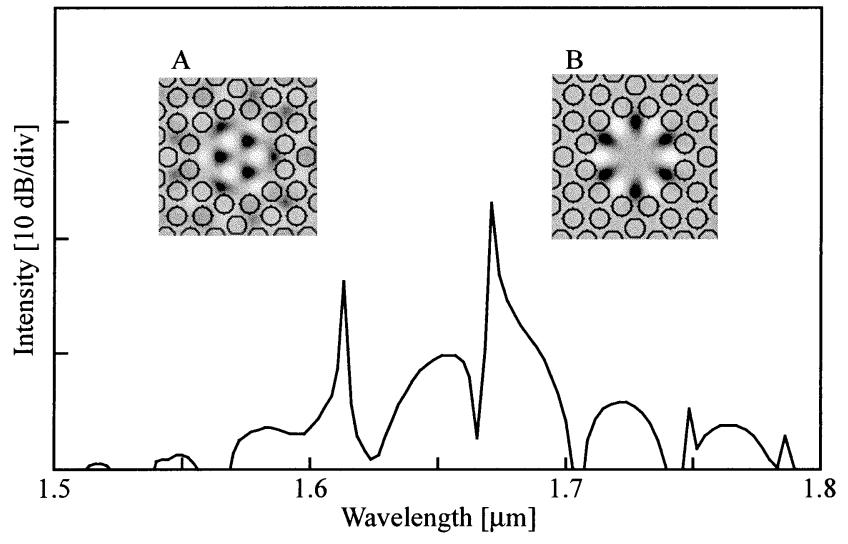

(b)

Fig. 8. Resonant spectra and $H_{z}$ distribution of each mode peak in 12-fold symmetric QPC with a point defect, which were calculated by the 2-D FDTD method. (a) Single missing hole. (b) Seven missing holes.

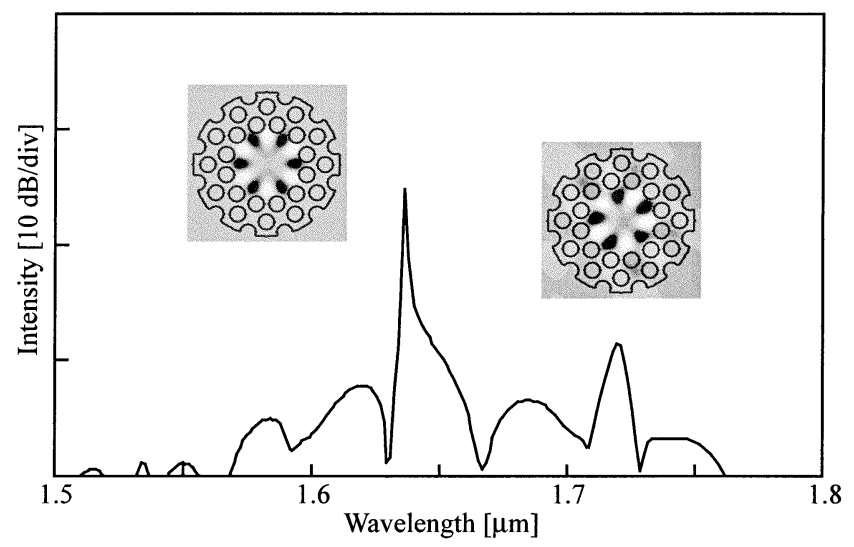

Fig. 9. Resonant spectra and $H_{z}$ distribution in the QPC gear, which is calculated by the 3-D FDTD method.

fined when it matches with the hole arrangement. The $Q$ factor of this mode was calculated to be 21600 , which is sufficiently high for lasing. Another broad peak at a longer wavelength is a mismatching mode having a smaller azimuthal order. It should be noted that in this structure, the $\pi$-phase-shifted mode is completely suppressed. The reason for this suppression is unclear, but it may be caused by the degeneracy similar to that in a microgear or a large phase shift of this mode by the QPC mirror, which disturbs the resonant condition in the defect. 


\section{CONCLUSION}

We fabricated microgear lasers of $\sim 2$ - $\mu$ m-diameter and 90-360 $\mathrm{nm}$ grating depth, and observed the room temperature $\mathrm{CW}$ lasing with the minimum threshold of $14 \mu \mathrm{W}$ and the single-mode operation in a deep grating device. The threshold is the lowest record for such kinds of microlasers. The results were well explained by the 3-D FDTD calculation and the surface recombination. In addition, we proposed the QPC gear, i.e., a fusion of a QPC point defect cavity and the microgear. The 2-D FDTD calculation demonstrated strongly localized modes in the QPC point defect cavity, which were similar to a dipole mode in a PC point defect cavity or a WGM in a microgear. The 3-D FDTD calculation of a more realistic device structure showed a high $Q$ factor being sufficient for lasing. We expect the a further update of the lowest threshold by the QPC gear with a limit diameter less than $1.6 \mu \mathrm{m}$ and with a passivation technique, [21] which reduces the surface recombination.

\section{ACKNOWLEDGMENT}

The authors would like to thank Prof. Y. Kokubun, Yokohama National University, and Prof. K. Iga, Prof. S. Arai, and Prof. F. Koyama, the Tokyo Institute of Technology, for helpful suggestions.

\section{REFERENCES}

[1] S. L. McCall, A. F. J. Levi, R. E. Slusher, S. J. Pearton, and R. A. Logan, "Whispering gallery mode microdisk lasers," Appl. Phys. Lett., vol. 60, pp. 289-291, 1992.

[2] A. F. J. Levi, S. L. McCall, S. J. Pearton, and R. A. Logan, "Room temperature operation of submicrometer radius disc laser," Electron. Lett., vol. 29, pp. 1666-1667, 1993.

[3] T. Baba, M. Fujita, A. Sakai, M. Kihara, and R. Watanabe, "Lasing characteristics of GaInAsP/InP strained quantum-well microdisk injection lasers with diameter of 2-10 $\mu \mathrm{m}$," IEEE Photon. Technol. Lett., vol. 9, pp. 878-880, 1997

[4] T. Baba, "Photonic crystals and microdisk cavities based on GaInAsP-InP system," IEEE J. Select. Topics Quantum Electron., vol. 3, pp. 808-830, 1997.

[5] J. P. Zhang, D. Y. Chu, S. L. Wu, S. T. Ho, G. Be, C. W. Tu, and R. C. Tiberio, "Photonic-wire laser," Phys. Rev. Lett, vol. 75, pp. 2678-2681, 1995.

[6] A. F. J. Levi, R. E. Slusher, S. L. McCall, S. J. Pearton, and W. S. Hobson, "Room-temperature lasing action in $\operatorname{In}_{0.51} \mathrm{Ga}_{0.49} \mathrm{P} / \mathrm{In}_{0.2} \mathrm{Ga}_{0.8} \mathrm{As}$ microcylinder lase diode," Appl. Phys. Lett., vol. 17, pp. 2021-2023, 1993.

[7] M. Fujita, R. Ushigome, T. Baba, A. Matsutani, F. Koyama, and K. Iga, "GaInAsP microcylinder (microdisk) injection laser with $\mathrm{AlInAs}\left(\mathrm{O}_{\mathrm{x}}\right)$ claddings," Jpn. J. Appl. Phys., vol. 40, pp. 5338-5339, 2001.

[8] M. Fujita, R. Ushigome, and T. Baba, "Strain relaxation effect in microdisk lasers with compressively strained quantum wells," Appl. Phys. Lett., vol. 80, pp. 1511-1513, 2002.

[9] - "Continuous wave lasing in GaInAsP microdisk laser with threshold of $40 \mu \mathrm{A}$," Electron. Lett., vol. 27, pp. 790-791, 2000.

[10] M. Fujita, K. Teshima, and T. Baba, "Low-threshold continuous-wave lasing in photopumped GaInAsP microdisk lasers," Jpn. J. Appl. Phys., vol. 40, pp. L875-L877, 2001.

[11] T. Baba, T. Hamano, F. Koyama, and K. Iga, "Spontaneous emission factor of a microcavity DBR surface emitting laser," IEEE J. Quantum Electron., vol. 27, pp. 1347-1358, 1991.

[12] M. K. Chin, D. Y. Chu, and S. T. Ho, "Estimation of the spontaneous emission factor for microdisk lasers via the approximation of whispering gallery modes," J. Appl. Phys., vol. 75, pp. 3302-3307, 1994.

[13] J. Vučković, O. Painter, Y. Xu, A. Yariv, and A. Scherer, "Finite-difference time-domain calculation of the spontaneous emission coupling factor in optical microcavities," IEEE J. Quantum Electron, vol. 35, pp. $1168-1175,1999$.

[14] M. Fujita, R. Ushigome, and T. Baba, "Large spontaneous emission factor of 0.1 in a microdisk injection laser," IEEE Photon. Technol. Lett., vol. 13, pp. 403-405, 2001
[15] M. Fujita and T. Baba, "Proposal and finite-difference time-domain simulation of whispering gallery mode microgear cavity," IEEE J. Quantum Electron, vol. 37, pp. 1253-1258, 2001.

[16] —-, "Microgear laser," Appl. Phys. Lett., vol. 80, pp. 2051-2053, 2002.

[17] M. E. Zoorob, M. D. B. Charlton, G. J. Parker, J. J. Baumberg, and M. C. Netti, "Complete photonic bandgaps in 12-fold symmetric quasi-crystals," Nature, vol. 404, pp. 740-743, 2000

[18] S. S. M. Cheng, L. M. Li, C. T. Chan, and Z. Q. Zhang, "Defect and transmission properties of two-dimensional quasi-periodic photonic band-gap systems," Phys. Rev. B, vol. 59, pp. 4091-4099, 1999.

[19] C. Jin, B. Cheng, B. Man, Z. Li, D. Zhang, S. Ban, and B. Sun, "Band gap and wave guiding effect in a quasi-periodic photonic crystal," Appl. Phys. Lett., vol. 75, pp. 1848-1850, 1999.

[20] T. Baba and D. Sano, "Low-threshold lasing and Purcell effect in microdisk lasers at room temperature," IEEE J. Select. Topics Quantum Electron., vol. 9, Sept./Oct. 2003.

[21] H. Ichikawa, K. Inoshita, and T. Baba, "Reduction in surface recombination of GaInAsP/InP micro-columns by $\mathrm{CH}_{4}$ plasma irradiation," Appl. Phys. Lett., vol. 78, pp. 2119-2121, 2001.

[22] O. J. Painter, R. K. Lee, A. Scherer, A. Yariv, J. D. O'Brien, P. D. Dapkus, and I. Kim, "Two-dimensional photonic band-gap defect mode laser," Science, vol. 284, pp. 1819-1821, 1999.

Kengo Nozaki was born in Niigata prefecture, Japan, on July 15, 1980. He received the B.E. degree, in 2003, from the Division of Electrical and Computer Engineering, Yokohama National University, Yokohama, Japan, where he is currently pursuing the M.E. degree.

Mr. Nozaki is a member of the Japan Society of Applied Physics (JSAP).

Atsuo Nakagawa was born in Chiba prefecture, Japan, on April 15, 1978. He received the B.E.degree, in 2002, from the Division of Electrical and Computer Engineering, Yokohama National University, Yokohama, Japan, where he is currently pursuing the M.E. degree.

Daisuke Sano was born in Shizuoka prefecture, Japan, on August 24, 1979. He received the B.E.degree, in 2002, from the Division of Electrical and Computer Engineering, Yokohama National University, Yokohama, Japan, where he is currently pursuing the M.E. degree.

Toshihiko Baba (M'93) was born in Nagano Prefecture, Japan, on November 12, 1962. He received the B.E., M.E., and Ph.D. degrees from the Division of Electrical and Computer Engineering, Yokohama National University (YNU), Yokohama, Japan, in 1985, 1987, and 1990, respectively.

During his Ph.D. work, he had been engaged in antiresonant reflecting optical waveguides (ARROWs) and lightwave circuits. In 1990, he joined the Tokyo Institute of Technology as a Research Associate. From 1991 to 1993, he worked on the spontaneous emission behavior in vertical cavity surface emitting lasers (VCSEL) and achieved the room temperature continuous wave (RT-CW) operation of a long-wavelength VCSEL. In 1994, he became an Associate Professor of YNU and started the research on photonic crystals (PCs), for which he reported the first fabrication and characterization of an InP-based light emitters and a line defect waveguide at lightwave frequencies, and microdisk lasers (MDLs), for which he achieved the first RT-CW operation with the smallest cavity and the lowest threshold, discussed some modifications of the cavity structure into microgear or PC-like structure, and demonstrated a MDL-based near field sensor. He also studied an enhancement of light-extraction efficiency and passivation techniques. His recent interests are in various cavities, lasers, and functional devices. He is also active on a deep-grating distributed Bragg reflector for short cavity lasers and Si photonics based on nanowire waveguides.

Prof. Baba is a member of the Institute of Electronics, Information and Communication Engineers (IEICE), the Japan Society of Applied Physics, and the American Physics Society. He received the Niwa Memorial Prize in 1991, the Best Paper Award of Micro-Optic Conference in 1993 and 1999, the Paper Award and Academic Encouragement Award from IEICE in 1994, and the Marubun Research Encouragement Award in 2000. 